\title{
CONSTRUÇÃO DE PROGRAMA DE SEGURANÇA DO PACIENTE EM HOSPITAL PÚBLICO DE ENSINO: PESQUISA DOCUMENTAL*
}

\author{
Denise Jorge Munhoz da Rocha ${ }^{1}$, Lillian Daisy Gonçalves Wolff ${ }^{2}$, Maria do Carmo Fernandez Lourenço \\ Haddad $^{3}$, Leila Soares Seiffert ${ }^{4}$, Otilia Beatriz Maciel da Silva ${ }^{5}$
}

\begin{abstract}
RESUMO: Pesquisa documental sobre a trajetória de um hospital público de ensino na construção de Programa de Segurança do Paciente. Foram consultados 200 documentos do período de 2004 a 2012, em um hospital público federal do sul do Brasil, com dados coletados entre maio de 2012 a abril de 2013. A análise documental evidencia estratégias essenciais à implantação de um programa. Entre elas, diagnóstico situacional com utilização de ferramentas da qualidade; auditorias internas com instrumentos customizados; profissionais com dedicação exclusiva e expertise na área da qualidade; capacitações direcionadas à equipe multiprofissional; incentivo à construção coletiva, o apoio da direção e o envolvimento de lideranças. O programa foi vanguardista entre os hospitais públicos federais. Desenvolvido previamente à legislação brasileira que normatiza a matéria, cumpriu em grande parte com as demandas da Resolução de Diretoria Colegiada n 36 de 25 de julho de 2013 do Ministério da Saúde.
\end{abstract}

DESCRITORES: Segurança do paciente; Qualidade da assistência à saúde; Gestão da qualidade.

\section{CONSTRUCTION OF A PATIENT SAFETY PROGRAM AT A PUBLIC TEACHING HOSPITAL: DOCUMENTARY RESEARCH}

ABSTRACT: Documentary research on the trajectory of a public teaching hospital in the construction of a Patient Safety Program Twohundred documents were consulted from the period between 2004 and 2012, at a federal public hospital in the South of Brazil, using data collected between May 2012 and April 2013. The documentary analysis evidences strategies essential for the implementation of a program. These include: situational diagnosis with use of quality tools; internal audits using customized tools; professional with exclusive dedication and expertise in the field of quality; multiprofessional team training; encouragement of collective construction, support from management and involvement of leaderships. The program was a forerunner among federal public hospitals. Developed before the Brazilian legislation that regulates the matter, the hospital complied with most of the requirements of Board Resolution 36 from July $25^{\text {th }} 2013$.

DESCRIPTORS: Patient safety; Quality of health care; Quality management.

\section{CONSTRUCCIÓN DE PROGRAMA DE SEGURIDAD DEL PACIENTE EN HOSPITAL PÚBLICO DE ENSEÑANZA: INVESTIGACIÓN DOCUMENTAL}

RESUMEN: Investigación documental acerca de la trayectoria de un hospital público de enseñanza en la elaboración de Programa de Seguridad del Paciente. Doscientos documentos fueron consultados en el periodo de 2004 a 2012, en un hospital público federal del sur de Brasil. Los datos fueron obtenidos entre mayo de 2012 y abril de 2013. El análisis documental muestra estrategias esenciales a la implantación de un programa. Entre ellas, la del diagnóstico situacional con utilización de herramientas de cualidad; auditorías internas con instrumentos customizados; profesionales con dedicación exclusiva y expertise en el área da cualidad; capacitaciones direccionadas el equipo multiprofesional; incentivo a la construcción colectiva, apoyo de la dirección y participación de liderazgos. El programa fue de vanguardia entre hospitales públicos federales. Desarrollado antes de la legislación brasileña que normatiza la materia, ayudó en las demandas de la Resolución de Directoría Colegiada n 36 de 25 de julio de 2013 del Ministerio e la Salud. DESCRIPTORES: Seguridad del paciente; Cualidad de la asistencia a la salud; Gestión de la cualidad.

*Artigo extraído da dissertação intitulada: “Gerenciamento de Risco em Hospital.” Universidade Federal do Paraná, 2013.

${ }^{1}$ Enfermeira. Mestre em Enfermagem. Chefe do Serviço de Vigilância em Saúde e Segurança do Paciente do Complexo do Hospital de Clínicas - Universidade Federal do Paraná. Curitiba, PR, Brasil.

${ }^{2}$ Enfermeira. Doutora em Engenharia de Produção. Docente da Universidade Federal do Paraná. Curitiba, PR, Brasil.

${ }^{3}$ Enfermeira. Doutora em Enfermagem Fundamental. Docente da Universidade Estadual de Londrina. Curitiba, PR, Brasil.

${ }^{4}$ Enfermeira. Doutoranda em Enfermagem. Universidade Federal do Paraná. Curitiba, PR, Brasil.

${ }^{5}$ Enfermeira. Mestre em Enfermagem. Chefe da Unidade de Gestão de Riscos Assistenciais do Complexo do Hospital de Clínicas - Universidade Federal do Paraná. Curitiba, PR, Brasil.

\section{Autor Correspondente:}

Denise Jorge Munhoz da Rocha

Universidade Federal do Paraná

R. Estevão Bayão, 200 - 80.240-260 - Curitiba, PR, Brasil

E-mail: denisejmr@uol.com.br
Recebido: 01/03/2016

Finalizado: 05/10/2016 


\section{- INTRODUÇÃO}

A partir do final dos anos 90, a área de segurança do paciente constitui-se uma das preocupações mundiais na área da saúde. O relatório To Err is Human: Building a Safer Health System (1999) ${ }^{(1)}$, estudo de maior impacto sobre o tema, trouxe à tona a dramática realidade dos incidentes de segurança do paciente relacionados à área da saúde.

Este primeiro relatório elevou os níveis de conhecimento sobre segurança na assistência em vários países, estimulou a conscientização por parte dos profissionais, acelerou as iniciativas governamentais com vistas à melhoria da segurança na assistência e deu origem a muitos outros estudos. Desde então, instituições privadas e governamentais de diversos países envidam esforços na elaboração e implantação de indicadores, metas nacionais de melhoria e programas voltados à segurança do paciente, por meio de campanhas educacionais ${ }^{(2-3)}$.

No referido relatório é proposta uma abordagem proativa, educativa e não punitiva das falhas, que estimule a análise multifatorial dos incidentes. O modelo sugerido transcende o tratamento superficial do erro, em que se elimina do cenário o que é mais visível e fraco, para investigar o que é mais profundo e difícil, ou seja, a abordagem sistêmica.

$\mathrm{Na}$ realidade brasileira, destaca-se no ano de 2002 a Rede Sentinela da Agência Nacional de Vigilância Sanitária (ANVISA) ${ }^{(4)}$, voltada à criação de um conjunto de hospitais que atuem como uma rede que notifica e toma medidas diante das suspeitas e problemas relacionados aos produtos na etapa pós-comercialização. Em 2011, é publicada a Resolução da Diretoria Colegiada - RDC nº 63 de 25 de novembro de $2011^{(5)}$, que dispõe sobre os Requisitos de Boas Práticas de Funcionamento para os Serviços de Saúde, e estabelece estratégias e ações voltadas à segurança do paciente.

O Programa Nacional de Segurança do Paciente (PNSP) foi lançado em abril de 2013, sustentado pela publicação da Portaria $n^{\circ} 529$ de $1^{\circ}$ de abril de 2013, do Ministério da Saúde. E em julho daquele ano, a ANVISA publicou a RDC n³6, de 25 de julho de 2013 que instituiu as ações para a segurança do paciente em serviços de saúde. As referidas legislações versam sobre o desenvolvimento de estratégias, produtos e ações direcionadas aos gestores, profissionais e usuários da saúde voltados à segurança do paciente, com o objetivo de mitigar a ocorrência de evento adverso na atenção à saúde ${ }^{(6-7)}$.

Percebe-se na realidade nacional uma lacuna desde o período da problematização do tema até o lançamento do Programa Nacional de Segurança do Paciente. No entanto, o conhecimento nessa área, gerado de forma dinâmica, pressionou as instituições a desenvolverem seus próprios métodos de trabalho em prol da segurança.

Na instituição na qual foi desenvolvida a pesquisa, as ações voltadas ao aprimoramento da qualidade têm como marco a sua missão declarada em 2002, na qual se comprometeu a prestar assistência acreditada à comunidade. Por conseguinte, em 2003 foi criada a Comissão de Acreditação Hospitalar, com o objetivo de implantar um Programa de Qualidade na organização, sendo necessário definir o método a ser seguido para aprimoramento da qualidade na assistência.

O presente estudo descreve a trajetória de construção de um programa de segurança do paciente em um hospital público de ensino.

\section{MÉTODO}

Trata-se de pesquisa documental. Este tipo de estudo utiliza-se de fontes diversificadas e dispersas. Os documentos constituem uma fonte rica e estável de dados, e como subsistem ao longo do tempo, são importantes fontes de dados nas pesquisas de natureza histórica. As pesquisas elaboradas a partir de documentos proporcionam melhor visão do problema ou hipóteses que conduzem a sua verificação por outros meios ${ }^{(8)}$.

A pesquisa documental envolveu as etapas descritas a seguir:

1) Determinação de objetivos: coleta de informações relacionadas à segurança do paciente; 
2) Elaboração do plano de trabalho:

2.1. Fontes de informação: definidos cinco tipos de documentos institucionais, sendo eles: 50 atas de reuniões da Comissão de Acreditação Hospitalar - CAH (de fevereiro de 2006 a maio de 2010); 22 atas do Comitê de Qualidade - CQ (de junho de 2010 a agosto de 2012) e 37 atas de reuniões internas da Assessoria de Gestão da Qualidade - AGQ (de junho de 2010 a maio de 2012); 09 planejamentos anuais da Comissão de Acreditação Hospitalar e do Comitê de Qualidade (2004 a 2012); Regimento Interno da AGQ; 81 roteiros de itens de verificação para as auditorias internas de qualidade; e cinco projetos de capacitações realizadas a partir de abril de 2010 a agosto de 2012, totalizando 200 documentos consultados. Estes documentos estavam arquivados na Assessoria de Gestão da Qualidade de um hospital público, federal, de ensino, voltado à assistência terciária, de grande porte, e localizado em uma capital no sul do Brasil.

2.2. Obtenção do material: os dados foram coletados no período de maio de 2012 a abril de 2013, por meio de leitura minuciosa dos documentos, nos quais foram identificados fragmentos que evidenciavam relação com o tema segurança do paciente;

2.3. Tratamento dos dados: foram selecionados os documentos referentes ao período de 2004 a 2012, os quais foram submetidos à análise temática, e foram constituídas seis categorias relativas às etapas da construção do Programa de Segurança do Paciente no hospital, a saber: diagnóstico preliminar da segurança do paciente; planejamento (primeira etapa); sensibilização/capacitação da comunidade hospitalar; planejamento (segunda etapa); notificação, investigação e monitoramento dos indicadores; e desenvolvimento de ciclo de melhoria.

2.4. Apresentação dos resultados: as fontes documentais foram codificadas com letras seguidas do número sequencial do documento, quando cabível, da sigla do local de origem do documento e do ano em que foi registrado. Os resultados foram discutidos tendo por base a legislação brasileira RDC $\mathrm{n}^{\circ} 36$, de 25 de julho de $2013^{(7)}$, referente à segurança do paciente.

Esta pesquisa documental foi aprovada em 16 de maio de 2012 pelo Comitê de Ética em Pesquisa em Seres Humanos da instituição, sob o registro Certificado de Apresentação para Apreciação Ética CAAE 02144512.0.0000.0096 e número de aprovação 22316.

\section{- RESULTADOS}

A trajetória de implantação do Programa de Segurança do Paciente, com a descrição sintetizada das atividades evidenciadas nos documentos, é apresentada no Quadro 1.

O hospital em estudo foi o primeiro entre os hospitais de ensino federais a obter certificação de acreditação pela Organização Nacional de Acreditação (ONA).

Quadro 1 - Síntese descritiva das principais estratégias realizadas no hospital no contexto do Programa de Segurança do Paciente, 2007 - 2012. Curitiba, PR, Brasil, 2013 (continua)

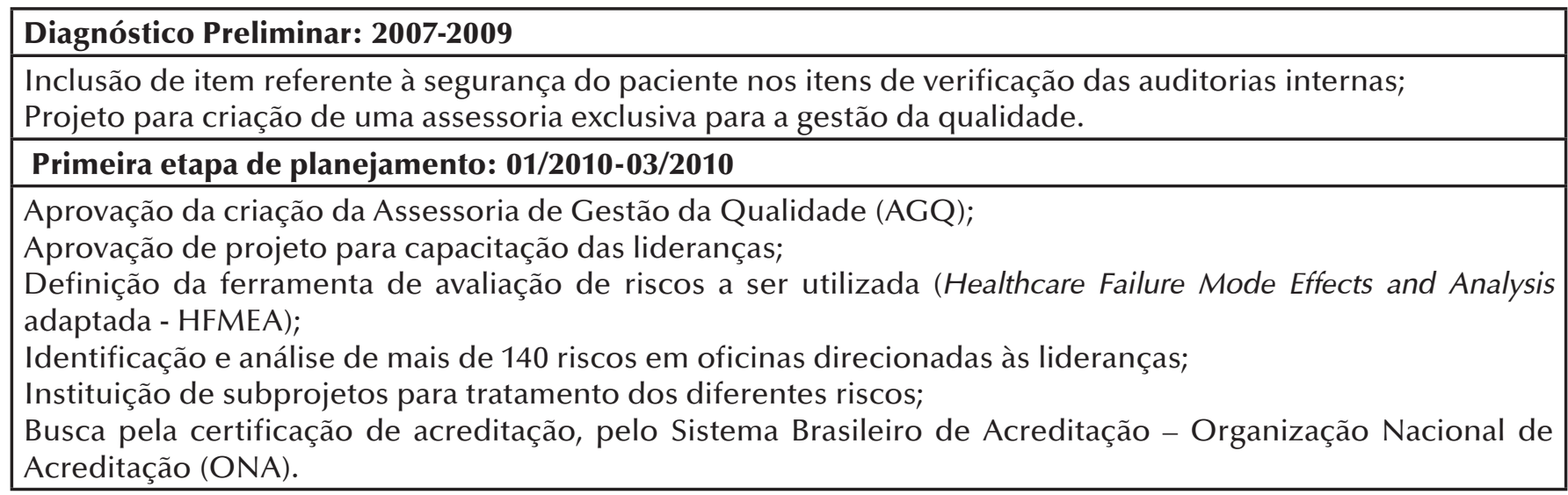




\section{Sensibilização/capacitação: 04/2010-08/2010; 02/2011-03/2011}

Realização de cursos e oficinas direcionados às lideranças, enfermeiros e grupos específicos, como equipes de apoio (nutrição, manutenção, recepção e outros);

Fórum aberto a toda comunidade hospitalar, enfocando as metas internacionais para a segurança do paciente e os 10 passos para segurança do COREN-SP (2010);

Capacitação para notificação de incidentes.

\section{Segunda etapa de planejamento: 08/2010-09/2010}

Desenvolvimento de ferramenta para notificação de incidentes;

Definição de método para análise de incidentes com grau de dano grave ao paciente - análise de causa raiz (ACR).

\section{Notificação/investigação/monitoramento de indicadores: 03/2011}

Definição de fluxos para tratamento de incidentes;

Início das notificações realizadas pela comunidade hospitalar;

Início das investigações de incidentes graves pela AGQ;

Definição de indicadores alinhados aos contratos de metas das unidades funcionais;

Definição das atribuições dos Grupos Internos da Qualidade (GIQ) para implementação de estratégias para melhoria da segurança do paciente.

\section{Desenvolvimento de ciclo de melhoria: 01/2012 - 08/2012}

Revisão dos formulários de notificação e investigação de incidentes;

Caracterização dos incidentes, conforme a International Classification for Patient Safety (ICPS), classe 1 - tipo de incidente;

Atualização e criação de procedimentos operacionais padrão - POP;

Atualização de fluxos de tratamento de incidentes;

Caracterização e disseminação do perfil de segurança institucional, com foco no quantitativo de notificações por unidade, tipo de incidente, grau de dano e recomendações de ações de melhoria;

Fórum das melhores práticas e desafios vivenciados pelos GIQ;

Organização acreditada em Nível I, pela ONA, busca a manutenção da certificação.

\section{- DISCUSSÃO}

O hospital de ensino em estudo planejou a implantação de um método que viabilizasse a melhoria da segurança na assistência ao paciente, fato que geralmente demanda mudança de cultura. Interpretase que essa ação representou um grande desafio, considerando o perfil da instituição: um hospital público de ensino. Autores reconhecem que em instituições desta natureza pode ocorrer um descaso com o bem público, tendo em vista que a estabilidade de emprego desestimula o profissional à melhoria e incentiva a acomodação ${ }^{(9)}$.

No entanto, observou-se que a construção do Programa de Segurança gerou uma intensa valorização da construção coletiva, com resultados que revelaram lideranças formais e profissionais comprometidos com a transformação da realidade.

Ressalta-se que as auditorias internas constituem importantes mecanismos de diagnóstico da realidade, que oportunizam o conhecimento de fragilidades e pontos fortes da organização, norteando de forma objetiva o desenvolvimento de ações de melhoria ${ }^{(9-10)}$.

Considera-se como um fator de impacto para evolução do programa, a criação de um departamento específico denominado Assessoria de Gestão da Qualidade (AGQ), diretamente subordinado à direção do hospital, com profissionais dedicados exclusivamente ao desenvolvimento de estratégias em prol da qualidade e segurança do paciente. Uma instituição que pretende implantar a gestão da qualidade tem como pré-requisito contar em seu quadro de recursos humanos com profissionais que se dediquem a esta função em tempo integral ${ }^{(10)}$.

A construção das planilhas Healthcare Failure Mode Effects and Analysis (HFMEA) pelas equipes multidisciplinares evidencia uma proposta de mudança de cultura pela comunidade hospitalar, com o abandono da passividade e assunção da proatividade, colaboração entre as diferentes áreas com participação das lideranças, e abordagem de riscos de forma sistêmica nas diferentes etapas do 
processo de trabalho ${ }^{(1,11-12)}$.

Em relação às capacitações, destaca-se o foco nas lideranças, ação institucional que corrobora com uma das premissas preconizadas pelo atual modelo de segurança do paciente, o qual destaca a importância do papel dos líderes na melhoria dos processos e das condições latentes que induzem às falhas e incidentes de segurança ${ }^{(1,11,13)}$.

Destaca-se que os profissionais da equipe de enfermagem são importantes agentes de segurança do paciente, permanecem junto a eles e seus familiares, tendo condições de avaliarem suas mudanças no momento em que ocorrem ${ }^{(13)}$. Além disso, são profissionais que permanecem vigilantes ao paciente, excedem os médicos na razão de ao menos 5:1 nas notificações de incidentes, e sentem-se muito mais confortáveis em discutirem as falhas com supervisores ${ }^{(14-15)}$.

A instituição em estudo adotou um sistema de notificação de incidentes, o que contribui para a captação de problemas relacionados à segurança do paciente. A autonotificação é o método mais comum para mensurar o nível de segurança ${ }^{(11)}$. No entanto, possui limitações e a baixa adesão dos profissionais é considerada a de maior impacto. Estudo americano comprovou que apenas 14\% dos eventos adversos são identificados por esse método, e apontou como motivo principal de subnotificação a dificuldade de percepção da equipe sobre o que é um incidente, e o que deve de fato ser notificado ${ }^{(15)}$.

Consequentemente, recomenda-se a mensuração do nível de segurança mediante a combinação de métodos com potencial para identificar erros ativos, eventos adversos e condições latentes para o erro $^{(11,16)}$.

A definição de estratégia para análise de incidentes graves constitui importante ação na gestão da qualidade e segurança. Ressalta-se que a análise de causa raiz é considerada um método que envolve a revisão interdisciplinar do incidente, com foco prioritário nos processos e sistema, o que previne a ênfase no fator humano como fator causal. O objetivo final é a identificação dos fatores contribuintes, que oportunizam o conhecimento da realidade, e subsidiam a proposta de mudanças para o aprimoramento do desempenho dos sistemas e a redução dos riscos de recorrência ${ }^{(17)}$.

A caracterização do perfil hospitalar relacionado ao quantitativo de incidentes reportados, ao grau de dano causado ao paciente e ao tipo de incidente, propiciam um diagnóstico situacional sistematizado, aprofundado, que viabiliza comparações tanto internas quanto externas à organização, e a impele à tomada de decisões ${ }^{(17)}$.

A certificação em qualidade constitui-se importante ferramenta de gestão, uma vez que se embasa em requisitos e padrões que norteiam a instituição à compreensão dos fatores para melhoria do seu desempenho, ao entendimento sistemático dos pontos fortes e oportunidades de melhoria, e a auxilia a promover a cooperação interna entre as diferentes áreas, processos e profissionais das equipes ${ }^{(18)}$.

A avaliação de acreditação estimula as organizações a constituir barreiras para prevenir, evitar e mitigar os riscos aos pacientes ${ }^{(19)}$.

\section{CONCLUSÃO}

Implantar um programa de segurança do paciente alinhado às estratégias definidas pela legislação brasileira requer conhecimento, foco, dedicação e persistência.

Organizações do porte do hospital em estudo são organismos vivos, nos quais os processos assistenciais não podem ser adiados, considerando que ocorrem conforme as necessidades se apresentam. Salienta-se, portanto, que o planejamento das ações é decisivo, e que cada instituição possui cultura e realidade próprias que devem ser consideradas. O modelo com maiores chances de sucesso é aquele com embasamento científico e adaptado, de forma participativa, à realidade institucional.

Destaca-se que mediante a análise dos documentos evidenciou-se que algumas estratégias foram essenciais à construção do Programa de Segurança do Paciente na instituição. Entre elas, o diagnóstico situacional com a utilização de ferramentas da qualidade; as auditorias com instrumentos customizados; 
os profissionais com dedicação exclusiva e expertise na área da qualidade; capacitações para a equipe multiprofissional; incentivo à construção coletiva e o envolvimento das lideranças formais e não formais em especial os da direção do hospital. Considera-se o processo de certificação em qualidade o fator de maior impacto positivo na implantação do programa.

O programa foi vanguardista entre os hospitais federais de ensino e foi desenvolvido previamente à legislação brasileira que normatizava a matéria. Já cumpriam em grande parte as demandas da RDC n³ 36 de 25 de julho de 2013 à época de sua construção.

No entanto, recomenda-se que se intensifiquem as estratégias que colocam o paciente na centralidade do cuidado; a adoção de outros métodos, além das notificações, para a identificação de incidentes; e a socialização das informações, em especial as que se referem ao aprendizado com o erro.

Nas instituições públicas, o desafio de construção de um Programa de Segurança do Paciente pode parecer maior, diante da premissa de que o progresso seja mais lento. Porém, estratégias adequadas podem viabilizar a obtenção de informações acerca dos profissionais e do ambiente em que desempenham suas atividades, a fim de que seja possível trabalhar as mudanças que são requeridas na organização hospitalar.

Apesar de o tema segurança do paciente gerar muitas publicações, é necessário aprofundar o conhecimento da realidade da segurança em organizações de saúde no Brasil e, nesse sentido, a recente legislação brasileira vem em auxílio para respaldar estratégias confiáveis a serem implantadas para a área de segurança do paciente.

\section{REFERÊNCIAS}

1. Kohn L, Corrigan J, Donaldson M. To Err Is Human: building a safer health system. Washington, DC: National Academy Press; 2000.

2. Zambon L. Pontos fundamentais para transformar a assistência em saúde em um processo seguro. Medicinanet. [Internet] 2010 [acesso em 26 abr 2016]. Disponível: http://www.medicinanet.com.br/conteudos/ gerenciamento/2719/como_transformar_a_assistencia_a_saude_em_um_processo_seguro.htm.

3. Leape L, Berwick D, Clancy C, Conway J, Gluck P, Guest J, et al. Transforming healthcare: a safety imperative. Qual Saf Health Care. 2009; (18): 424-8.

4. Agência Nacional de Vigilância Sanitária. Rede Sentinela. [Internet] Brasília: Agência Nacional de Vigilância Sanitária; 2009 [acesso em 10 jan 2016]. Disponível:

http://www.anvisa.gov.br/servicosaude/hsentinela/historico.htm

5. Ministério da Saúde (BR). Resolução da Diretoria Colegiada RDC n. 63, de 25 de novembro de 2011. Dispõe sobre os requisitos de boas práticas de funcionamento para os serviços de saúde. Diário Oficial da União, Brasília, 28 nov. 2011. Seção 1.

6. Ministério da Saúde (BR). Gabinete do Ministro. Portaria n. 529, de $1^{\circ}$ de abril de 2013. Institui o Programa Nacional de Segurança do Paciente (PNSP). Diário Oficial da União, Brasília, 02 abr. 2013. Seção 1.

7. Ministério da Saúde (BR). Agência Nacional de Vigilância Sanitária. Resolução da Diretoria Colegiada - RDC n. 36, de 25 de julho de 2013. Institui ações para a segurança do paciente em serviços de saúde e dá outras providências. Diário Oficial da União, Brasília, 26 jul. 2013. Seção 1.

8. Gil AC. Como elaborar projetos de pesquisa. $3^{a}$ ed. São Paulo: Atlas; 1991.

9. Paladini EP. Gestão da Qualidade: teoria e prática. 2ª ed. São Paulo: Atlas; 2010.

10. Falconi V. O verdadeiro poder. Nova Lima: INDG; 2009.

11. Wachter RM. Compreendendo a segurança do paciente. Porto Alegre: Artmed; 2013. 
12. Feldman LB. Gestão de risco e segurança hospitalar. São Paulo: Martinari; 2008.

13. Joint Comission Resources. Temas e estratégias para liderança em enfermagem: enfrentando os desafios hospitalares atuais. Porto Alegre: Artmed; 2008.

14. Wild D, Bradley E. The gap between nurses and residents in a community hospital's error-reporting system. Jt Comm J Qual Patient Saf. 2005; 31(1): 13-20.

15. Department of Health and Human Services (US). Hospital incident reporting systems do not capture most patients harm. [Internet] 2012 [acesso em 26 abr 2016]. Disponível:

https://oig.hhs.gov/oei/reports/oei-06-09-00091.pdf.

16. Thomas EJ, Petersen LA. Measuring errors and adverse events in health care. J Gen Intern Med. 2003;18(1):617.

17. World Health Organization (WHO). Conceptual framework for the international classification for patient safety. [Internet] Geneve: WHO; 2009 [acesso em 10 jul 2015]. Disponível:

http://www.who.int/patientsafety/taxonomy/icps_full_report.pdf.

18. Organização Nacional de Acreditação (ONA). Manual das organizações prestadoras de serviços de saúde. Brasília: Organização Nacional de Acreditação; 2014.

19. Seiffert LS, Wolff LDG, Wall ML. A expertise de Nightingale e o manual brasileiro de acreditação de organizações de saúde. Cogitare enferm. [Internet] 2011; 16(3) [acesso em 22 fev 2016]. Disponível:

http://dx.doi.org/10.5380/ce.v16i3.24293. 